\title{
Sobre la relación regla/excepción. Una aproximación inferencial
}

\author{
Federico León Szczaranski Vargas*
}

\begin{abstract}
RESUMEN
El artículo ofrece una reconstrucción de la relación reglalexcepción en consideración a las relaciones inferenciales materiales entre ambas. Para ello y siguiendo a Luis Duarte D'Almeida, primero se examinarán tres aproximaciones a esta relación y se identificarán dos constreñimientos que sirven como estándares de corrección a los que ellas se han de adecuar. Luego y recurriendo a la semántica inferencial de Robert Brandom, se propondrá una nueva variante de la aproximación "conflictual" al problema que, reconociendo la existencia de un conflicto normativo entre regla y excepción, logra no solo adecuarse a los estándares identificados por Duarte, sino que posibilita desactivar el conflicto recurriendo exclusivamente a las reglas involucradas.
\end{abstract}

Regla y excepción; conflictos normativos; semántica inferencial

\section{On the ruleeexception relation. An inferential approach}

\begin{abstract}
The paper offers a reconstruction of the rulelexception relation grounded in the material inferential relations between them. To this end and following Luis Duarte D"Almeida, three approaches to this relation are analysed and two constrains that serve as correction standards for them are identified. Then and resorting to Robert Brandom"s inferential semantics, a novel variant of the "conflictual" approach is proposed, one that recognising a normative conflict between rule and exception, manages to satisfy Duarte"s standards and deactivate the conflict resorting only to the involved rules.
\end{abstract}

Rule and exception; normative conflicts; inferential semantics

* Licenciado en Ciencias Jurídicas y Sociales, Universidad de Chile. Doctor en Derecho, Universidad de Glasgow, Escocia. Profesor de Derecho, Universidad Central, Chile. ORCID: https://orcid.org/00000001-6207-8709. Correo electrónico: federico.szczaranski@ucentral.cl

Artículo recibido el 26.9.20 y aceptado para su publicación el 8.1.21. 


\section{INTRODUCCIÓN}

l inicio de Allowing for Exceptions, Luis Duarte afirma que "la línea entre aque-
llo que cuenta como una excepción y aquello que es parte de una regla es más
difícil de dibujar de lo que parece"l. En ningún caso se trata de un juicio original, pero ello no obsta a que Duarte, en su intento por ofrecer una reconstrucción de la relación entre regla y excepción que supere los defectos que él adscribe a las formas tradicionales en la que ella ha sido articulada, identifique adecuadamente dos condiciones que cualquier teoría abocada a dibujar dicha línea debe satisfacer para poder ser aceptada. A saber, la explicación de la relación entre regla y excepción debe, por una parte, permitir una especificación de las condiciones suficientes para la corrección de una decisión judicial, lo que sirve para defender la idea con las que las decisiones legales pueden ser presentadas como siguiéndose deductivamente de alguna combinación de enunciados acerca de reglas y hechos del caso $^{2}$. Por otra parte, hacer justicia a la idea de que "hay una distinción importante entre excepciones y negación de los elementos ordinarios de una regla" 3 , lo que sirve para defender la intuición de que la ausencia de -por ejemplo- legítima defensa en un caso de homicidio es en algún sentido relevante distinta a la ausencia del consentimiento en un caso de violación ${ }^{4}$.

En lo que sigue se propondrá una nueva aproximación a la relación entre regla y excepción ${ }^{5}$, una que no solo se adecue a los constreñimientos identificados por Duarte, sino que también pueda superar las deficiencias del modelo por él propuesto, reinterpretando su principal conclusión desde una perspectiva "expresiva”. Para ello, recurriendo a la semántica inferencial de Robert Brandom, la relación regla/excepción será articulada en función de las relaciones "materiales" entre los contenidos proposicionales de ambas.

\section{INCORPORACIONISMO, NO DEDUCTIVISMO Y PROCEDIMENTALIZACIÓN}

El primer paso en la elaboración de este nuevo modelo consiste en precisar la forma en que operan los constreñimientos identificados por Duarte, y para ello resulta conveniente analizar cómo las aproximaciones tradicionales que él enfrenta no logran (según él) adecuarse a estos. Un caso de operación no problemática de legítima defensa servirá para ejemplificar el problema: supongamos que $\mathrm{K}$ se apresta a disparar ilegítimamente al cuerpo de J, quien -para repeler el ataque- lo apuñala causándole la muerte. Si la puñalada de J sirvió como acción racionalmente necesaria para impedir el disparo, entonces

\footnotetext{
${ }^{1}$ Duarte, 2015 a, p. 5.

${ }^{2}$ En contra de la fórmula tradicional del silogismo: Duarte, 2019.

${ }^{3}$ Duarte, 2015 a, p. 6.

${ }^{4}$ Duarte, 2015a, p. 6.

${ }^{5}$ Al elaborar su teoría, Duarte distingue entre excepciones explícitas e implícitas (al respecto, véase MacCormick, 1995, p. 99). El presente trabajo se abocará solo a las primeras.
} 
esta puede ser verdaderamente descrita como "matar a K en legítima defensa". Bajo esta descripción, al caso le serían en principio aplicables tanto la disposición que sanciona el homicidio como aquella que exime de toda sanción a quien actúe en legítima defensa. Si bien hay acuerdo en solucionar la incompatibilidad entre las consecuencias establecidas por cada disposición por la vía de reconocer carácter de excepción a la circunstancia consistente en actuar en legítima defensa, es en relación con la forma de dar cuenta tal carácter excepcional que las aguas se dividen.

\section{La vía incorporacionista}

Aunque pareciera natural admitir la existencia de reglas que entran en conflicto en casos como los de legítima defensa, la corriente que Duarte califica de "incorporacionista" afirma, por el contrario, que al respecto "ningún jurista aceptaría que el sistema genera contradicciones" 6 , por cuanto "los conflictos de normas (...) pueden y deben ser solucionados mediante interpretación" ${ }^{7}$. En el modelo kelseniano ${ }^{8}$, el fundamento para tal operación interpretativa consiste en entender que, en tanto norma dependiente, la excepción de legítima defensa opera "limitando la esfera de validez" de la norma que castiga matar a otro 9 . No habría diferencia entre especificar una cualidad de una determinada clase en la definición de la clase misma y especificar dicha cualidad como parte del contenido de una excepción a la clase ${ }^{10}$. Entonces la noción misma de "excepción" resultaría "lógicamente vacía"11: correspondería a una "construcción lingüística" cuyo rol se reduciría a establecer los límites de la regla ${ }^{12}$. Ello permitiría sostener que, a pesar de las apariencias, las disposiciones en conflicto no dan lugar a dos reglas cuyos consecuentes son incompatibles ${ }^{13}$, sino que ambas forman una unidad, una única regla que sanciona matar en supuestos que no constituyen, también, una instancia de legítima defensa. Al tomar en consideración las demás disposiciones que establecen circunstancias en las que no corresponde aplicar pena alguna, resulta posible obtener una completa formulación de la regla que castiga el homicidio por la vía de incluir en su antecedente la negación de la disyunción de dichas circunstancias ${ }^{14}$.

La objeción comúnmente levantada en contra de esta alternativa dice relación con la inconsistencia en la que se incurre al afirmar, por una parte, que en el sistema no se

${ }^{6}$ Rodríguez, 1997, p. 159.

${ }^{7}$ Kelsen, 1991, p. 45 y 2005, p. 207.

${ }^{8}$ Modelo caracterizado por postular el principio de no contradicción en todo sistema jurídico, KELSEN, 2006, p. 406.

${ }^{9}$ Kelsen, 2005, p. 55; y antes Bentham, 1970, pp. 113 y ss.

${ }^{10}$ STONE, 1944, p. 280; 1985, pp. 68-73.

11 Schauer, 1991, p. 893.

12 Williams, 1988, p. 262.

${ }^{13}$ Respecto del razonamiento moral esta es la opción favorecida por Moore, 1989a, p. 847 y 1989 b, p. 315. En contra: Raz, 1989, p. 1155.

${ }^{14}$ Williams, 1982; Waluchow, 2012, p. 260. 
verifican conflictos; y por otra, que estos últimos han de ser resueltos mediante un ejercicio interpretativo $^{15}$-se estaría así ilegítimamente infiriendo la inexistencia de conflictos a partir de la posibilidad de solucionarlos ${ }^{16}$-. En contra de lo sostenido originalmente por Kelsen, cabría replicar que "si las contradicciones deben ser eliminadas, entonces debe haber algo así como una contradicción que debe ser eliminada" 17 .

Ante esta observación, la corriente incorporacionista habría de responder indicando que el conflicto al que se hace referencia es uno potencial ${ }^{18}$, advertido al atender a las formulaciones normativas ${ }^{19}$. Si las disposiciones legales en cuestión fueran tenidas, cada una, como formulación de una regla distinta ${ }^{20}$, entonces se configuraría un conflicto normativo entre ellas, y es justamente la prevención de este último lo que permite identificar el contenido de la regla resultante ${ }^{21}$ : la razón para incluir a la circunstancia tenida como excepcional como un elemento negativo en el antecedente de la regla, es que de lo contrario se arribaría a conclusiones incompatibles para el mismo caso.

Con independencia de qué tan convincente resulte esta respuesta, resulta fundamental notar que la alternativa incorporacionista se encuentra en una posición en la que resulta particularmente difícil explicar el carácter mismo de "excepción” que exhibirían las circunstancias tenidas por excepcionales: si bien el punto de partida en el que se construye el modelo consiste en reconocer a ciertas circunstancias como "excepciones", al incluir a la negación de estas en el antecedente de las reglas que deben ser aplicadas, dichas circunstancias ya no pueden ser consideradas como excepciones a aquellas mismas reglas. Si la regla que enuncia las condiciones para ser elegido congresista dispusiera que "el candidato que obtenga más votos en su circunscripción y que no sea dirigente sindical ocupará el escaño respectivo", entonces "ser dirigente sindical” no podría constituir una excepción a dicha regla. Para que una determinada circunstancia pueda constituir una excepción a una regla parece necesario que esta última abarque casos en los que la circunstancia excepcional se verifica, de forma tal que, de no reconocerse la excepción, a dichos casos correspondería asignarles la solución prevista por la regla en cuestión ${ }^{22}$. Es justamente esto lo que se ve imposibilitado en caso de seguirse la alternativa incorporacionista, ya que los casos de dirigentes sindicales no instancian los hechos operativos de la regla, entonces respecto de ellos no hay espacio para reconocer el "ser dirigente sindical" como una excepción - una excepción, para ser tal, tendría que ser "externa” a la regla respecto de la cual ella es una excepción ${ }^{23}-$.

\footnotetext{
${ }^{15}$ Así Rodríguez, 1997, p. 159.

${ }^{16}$ RAZ, 1980, p. 224.

17 Alchourrón y Bulygin, 1981, p. 113. En sentido similar: Ortiz de Urbina, 2008, p. 24; MaÑALiCH, 2014b, p. 479.

18 RODRÍGUEZ y SUCAR, 1998, pp. 407 y 410.

19 Von Wright, 1963, Capítulo VI. Cf. Ferrer y Rodríguez, 2011, p. 164.

${ }^{20}$ Acerca de la relación entre disposiciones y normas: GUASTINI, 1999, pp. 100 y ss.

${ }^{21}$ En sentido similar: SEARLE, 1987, pp. 84-85.

22 Así Schauer, 1991, p. 874; Miller, 1956, p. 262.

23 Duarte, 2015a, p. 4, 153; Finkelstein, 1999, pp. 507-508.
} 
Esta corriente logra entonces satisfacer el primer constreñimiento identificado por Duarte, por cuanto el contenido de la regla resultante especifica las condiciones suficientes para la corrección de una decisión que imponga la consecuencia de la regla, pero lo hace al costo de equiparar a la verificación de una circunstancia considerada excepcional con la verificación de un elemento negativo ordinario de la regla, lo que a su vez imposibilita reconocer el carácter excepcional de dicha circunstancia ${ }^{24}$. La objeción contra esta aproximación, empero, no supone negar que el resultado al que se debe arribar sea exactamente el mismo tanto en los casos en los que se verifica una circunstancia excepcional como en aquellos en los que se verifica un elemento negativo ordinario de la regla (o en los que no se verifica un elemento positivo de ella). Más bien, de lo que se trata es de reconocer que, aun cuando en ambos supuestos la conclusión a ser alcanzada es la misma, la forma en la que excepciones y antecedentes de hecho de una regla contribuyen a la corrección de la decisión es diferente ${ }^{25}$. Es esa diferencia la ignorada por la corriente incorporacionista, para estos tratar casos de dirigentes sindicales como excepciones a una regla que dispone (simplemente) que el candidato más votado ocupará el escaño respectivo, sería indistinguible de tratar a "no ser dirigente sindical" como un elemento de la regla que establece las condiciones para ocupar escaños ${ }^{26}$.

\section{La vía no-deductivista}

La posibilidad de reconocer a ciertas circunstancias como "excepciones”, asignándoles un rol distinto al que caracteriza a la operación de los elementos negativos ordinarios incluidos en los antecedentes de las reglas, se ve favorecida por la adopción del modelo que Duarte denomina "no-deductivista”. Acá, el antecedente de la regla que determina las condiciones bajo las cuales un escaño puede ser ocupado solo hace referencia a haber obtenido más votos; y si bien la satisfacción de dicho antecedente permite concluir que quien lo instancia habrá de ocupar el escaño respectivo, esta inferencia es derrotable en atención a la calidad de dirigente sindical que puede exhibir un candidato. Esto último equivale a asignar carácter no-monotónico ${ }^{27}$ al razonamiento legal: las conclusiones que correctamente se derivan a partir de un set de reglas pueden ser derrotadas si el set se expande incluyendo a una excepción. De esta forma, el modelo es no-deductivista precisamente porque la verificación del antecedente de la regla constituye una condición necesaria, pero solo suficiente para la corrección de la conclusión establecida por esta en caso que no se verifiquen las circunstancias excepcionales.

${ }^{24}$ Duarte, 2015 b, p. 39.

${ }^{25}$ Si bien el punto será abordado detalladamente más adelante, por ahora vale la pena hacer presente que la importancia de esta diferencia es inmediatamente reconocible al notar que, de equiparar excepciones y elementos ordinarios, no parece posible explicar, por ejemplo, por qué no es en principio necesario probar la ausencia de legítima defensa para poder arribar a una condena por homicidio; pero sí es necesario probar la ausencia de consentimiento para lograr una condena por violación.

26 Williams, 1988, p. 278.

${ }^{27}$ Brandom, 2014, p. 20. 
Identificar la particular forma de operación que conforme a un modelo no-deductivista caracteriza a las circunstancias excepcionales y que permite distinguirlas de aquellas consideradas ordinarias, se ve facilitado al advertir que, aun cuando la manera en la que Duarte construye la distinción entre "incorporacionistas" y "no-deductivistas" no descansa en que los primeros nieguen y los segundos afirmen que en estos casos se configure un "conflicto normativo", este último modelo sí ofrece espacio para reconocer la existencia de tal conflicto. Si el contenido del antecedente de nuestra regla electoral es “el candidato que obtenga más votos en su circunscripción ocupará el escaño respectivo", y se quiere sostener que dicha conclusión no ha de ser alcanzada respecto de dirigentes sindicales, la manera obvia de alcanzar dicho objetivo es asumiendo una segunda regla cuyo antecedente abarque los casos de estos últimos, impidiendo respecto de ellos la imposición de la consecuencia establecida por la primera -y habrían dos reglas que pueden ser formuladas para lograr este propósito ${ }^{28}$.

En primer término, es posible entender que la circunstancia tenida por excepcional es parte del presupuesto de hecho de alguna otra regla cuyo consecuente es incompatible con la primera. Así, junto con la regla electoral indicada, se reconocería una segunda regla en la que, por ejemplo, "los dirigentes sindicales no ocuparán ningún escaño". De esta forma, en un caso que caiga dentro del rango inmediato ${ }^{29}$ de ambas se configurará un conflicto normativo ${ }^{30}$ cuya solución pasará por la invocación de una regla de preferencia ${ }^{31}$ que, sirviendo para distinguir entre aplicabilidad interna y externa de una regla ${ }^{32}$, lo desactiva mediante el desplazamiento de una de las reglas que lo genere, determinando así que solo la otra debe ser aplicada ${ }^{33}$. La segunda forma que podría asumir una regla cuya aplicación podría impedir, respecto de dirigentes sindicales, que se alcance la conclusión establecida por la regla electoral, sería una que dispusiera que respecto de ellos esta última regla no es aplicable -en este caso, esta segunda regla sería ella misma una regla que opera desplazando a la regla electoral, afectado así su aplicabilidad externa sin entrar en conflicto con ella-. Mientras que la primera variante será denominada "conflictual", la segunda será llamada "no-conflictual".

28 Ortiz de URbina, 2008, p. 19

${ }^{29}$ RAZ, 1980, p. 123.

${ }^{30}$ Ross, 1959, p. 128; Von Wright, 1983, p. 156 y 1991, p. 271; Alchourrón y Bulygin, 1981, p. 112; Hamblin, 1972, p. 74; Elhag, et al., 2000, pp. 207-217; Kelsen, 1991, p. 123; Sartor, 1992 , p. 209; HaMMER, 1987, p. 230.

31 Alchourrón y Bulygin, 1981, p. 107. Una vez que Kelsen pasa a aceptar conflictos entre reglas válidas, entiende también que ellos solo pueden ser resueltos por una tercera regla, KELSEN, 1973, p. 124. Usando una terminología distinta: Weinberger, 1972, p. 325; Von Wright, 1991, p. 277. Distinguiendo entre aserciones de preferencia y reglas de aplicabilidad, SARTOR, 1994, pp. 184 y 190.

32 Navarro y Moreso, 1997; Navarro et. al., 2004. De la manera en que acá son entendidas, las reglas de preferencia, a diferencia de los "principios de derogación" de Kelsen, solucionan el conflicto sin afectar la validez de las reglas que lo generan.

33 Navarro y Moreso, 1997, p. 203 y 209; Navarro y Rodríguez, 2014, p. 129; Bulygin y Mendonca, 2005, pp. 69. 
Ninguna de estas alternativas, sin embargo, es analizada por Duarte. La forma de no deductivismo que él considera es aquella que hiciera célebre H.L.A. Hart en The Adscription of Responsibility and Rights ${ }^{34}$ : justamente para negar que las circunstancias excepcionales puedan ser reducidas a condiciones ordinarias mediante la inclusión de su negación en el antecedente de las reglas respectivas, y así sostener que, habiendo derrotabilidad de por medio, no es posible ofrecer un set de condiciones necesarias y suficientes para la corrección de una decisión (en los términos aquí utilizados: para rechazar el modelo "incorporacionista") ${ }^{35}$; la opción favorecida por Hart para dar cuenta del carácter excepcional de estas circunstancias descansaría en la introducción de una cláusula "a menos que". Incluyéndose esta, las condiciones ordinarias establecidas para una conclusión permiten arribar a ella "a menos" que en el caso particular "otras circunstancias estén presentes las cuales lo lleven bajo una excepción reconocida" 36 . La manera en que Duarte reconstruye la variante hartiana de no-deductivismo pasa entonces por incluir en la regla en cuestión una "cláusula excepcional" 37 referida a esas circunstancias y ubicada a continuación de su consecuente. Así, la variante hartiana de la regla electoral sería la siguiente: "el candidato que obtenga más votos en su circunscripción ocupará el escaño respectivo, a menos que se trate de un dirigente sindical” 38 .

Estas tres formas de "no-deductivismo" suponen una regla cuyo antecedente está integrado solo por la circunstancia consistente en ser el candidato más votado, por lo que en un caso que verifique dicha propiedad sería correcto arribar la conclusión por ella establecida -que tal candidato ocupará el escaño respectivo-. No obstante, esta conclusión será derrotada en aquellos casos en los que también se verifique la circunstancia tenida por excepcional, i.e., ser dirigente sindical, circunstancia que corresponderá o bien al antecedente de una regla "preferida" (en la variante conflictual), o bien al antecedente de una regla de preferencia (en la variante no-conflictual), o bien al contenido de una cláusula excepcional respecto del ámbito de aplicación de la regla misma (en la variante hartiana). Así, a diferencia del modelo "incorporacionista”, aun cuando la verificación de la circunstancia excepcional constituye una condición suficiente para que no se pueda arribar a la conclusión en cuestión, su no verificación no constituiría una condición necesaria para poder correctamente alcanzarla: solo "ser el candidato que ha obtenido más votos” exhibiría el carácter de condición necesaria, siendo también suficiente en caso de no verificarse la circunstancia excepcional.

Distinguiendo entre condiciones necesarias para la conclusión y suficientes para derrotarla, este modelo permitirá diferenciar la forma en que operan elementos ordinarios y excepciones: mientras que los primeros constituyen circunstancias que "deben verificarse” para que la conclusión en cuestión pueda correctamente ser alcanzada, los

${ }^{34}$ Hart, 1949. Acerca de derrotabilidad y textura abierta, fundamental: STAVropoulos, 1996, Capítulo III. Recientemente: García, 2020.

35 HART, 1949, p. 174.

36 HART, 1949, p. 174.

${ }^{37}$ RAZ, 1980, p. 56.

38 Duarte, 2015 a, p. 17. 
segundos corresponden a circunstancias que "si se verifican, derrotan" dicha conclusión 39 -es esta diferencia la que debe ser defendida para evitar colapsar ambas categorías-.

A pesar de que Duarte solo considera la variante hartiana, la objeción por él levantada parece ser extensible también a los otros dos supuestos de no-deductivismo, y es que en estas tres formulaciones la verificación de la circunstancia excepcional es tenida por suficiente para impedir que se pueda correctamente arribar a la conclusión especificada por el consecuente de la regla, y de ello se seguiría, al fin y al cabo, que la ausencia de dicha circunstancia habría de contar como condición necesaria para la corrección de la decisión en cuestión ${ }^{40}$ : si ser dirigente sindical es suficiente para no ocupar el escaño respectivo, entonces la ausencia de dicha propiedad es condición necesaria para ocupar$10^{41}$. Si bien esta inferencia será derrotada más adelante, lo que importa ahora es atender a la conclusión a la que Duarte llega a partir de ella, a saber, que resulta inevitable, partiendo del modelo no-deductivista, terminar arribando igualmente a la conclusión que es defendida por el modelo incorporacionista: la no verificación de la conjunción de las excepciones ocupa el mismo "estatus lógico" que la verificación de las circunstancias tenidas por ordinarias ${ }^{42}$, de forma tal que ambas especifican condiciones necesarias para la corrección de la decisión ${ }^{43}$. Y el problema es que entonces se pierde de vista que excepciones y elementos ordinarios constreñirían de maneras distintas la corrección de la decisión. Aunque lleven al mismo resultado, hay una diferencia jurídicamente relevante entre afirmar que un sujeto no ha matado y que lo ha hecho en legítima defensa.

\section{Inclusión de consideraciones procedimentales}

La vía elegida por Duarte para intentar satisfacer ambas condiciones pasa por adoptar una postura intermedia entre las aproximaciones mencionadas: alineándose con la corriente incorporacionista y en contra la no-deductivista, su propuesta consiste en afirmar que regla y excepción forman ambas una única regla, en cuyo antecedente se encuentran incorporadas aquellas circunstancias que son tenidas como excepciones. Sin embargo, alineándose con la corriente no-deductivista y contra la incorporacionista, dicha incorporación es efectuada mediante una formulación que posibilita reconocer la diferencia entre excepciones y elementos ordinarios. De esta manera, intenta que el contenido de esta única regla sea tal que permita tanto especificar las condiciones suficientes para la corrección de una decisión en que se afirma su consecuente, como reconocer la diferencia entre circunstancias excepcionales y comunes.

Para ello, Duarte comienza sosteniendo que en un contexto judicial "no podemos simplemente decir que una circunstancia X 'está presente' si es el caso que X, y que 'está

\footnotetext{
${ }^{39}$ DuARTe, 2015a, p. 15.

${ }^{40}$ En este sentido, véase: BAKER, 1977, p. 34.

41 Duarte, 2015 a, p. 49.

42 Duarte, 2015 a, p. 50.

43 Mackie, 1985 , p. 32.
} 
ausente' si no es el caso que X" ${ }^{44}$. Lo relevante, en cambio y siguiendo a Kelsen ${ }^{45}$, es si puede decirse que dicha circunstancia ha sido "probada" conforme con ciertas reglas y procedimientos. Considerando esta dimensión procedimental de la verificación de las circunstancias ordinarias y excepcionales resulta posible dar cuenta en el contenido mismo del antecedente de la regla a ser aplicada de las diferentes formas en que unas y otras operan. Las circunstancias ordinarias "deben verificarse" para que se pueda alcanzar la conclusión en cuestión, lo que no sería adecuadamente expresado por la mera inclusión de dicha circunstancia en el antecedente de la regla ${ }^{46}$. Más bien, para que los hechos operativos puedan dar cuenta de esta dimensión procedimental, ellos deben formularse haciendo referencia explícita a la necesidad de que la circunstancia sea probada. Y es mediante esta explicitación que Duarte busca salvar el aparente problema advertido respecto de las variantes no-deductivistas: en todas ellas, la verificación de la circunstancia excepcional cuenta (correctamente) como condición suficiente para que la conclusión no pueda ser alcanzada; pero ello entrañaría que la ausencia de dicha circunstancia constituiría (incorrectamente y tal como la verificación de las circunstancias ordinarias) una condición necesaria para arribar a aquella. De aceptarse esta equiparación, entonces a la conclusión establecida por la regla solo podría arribarse una vez que, junto con probarse las circunstancias ordinarias, se prueba también la no verificación de las circunstancias consideradas excepcionales.

Esta equiparación en el modo de operar de excepciones y elementos ordinarios es evitada al precisar que, respecto de aquellas, lo que es necesario para la corrección de la conclusión no es que sea probado que estas no se verifican, sino que ellas "no deben probarse" 47 - esta sería la forma adecuada de incluir en el antecedente de la regla a la "negación" de las circunstancias excepcionales-. Así, si "O" representa a las circunstancias ordinarias, "E" a las excepcionales y "C" a la conclusión, entonces la regla puede expresar que las primeras deben "estar presentes” y las segundas "ausentes” mediante la siguiente formulación: "si es probado que $\mathrm{O}$ y no es probado que E entonces C". Y una vez que estas diferentes formas de operación han sido notacionalmente codificadas y expresadas en el contenido de la regla, entonces esta última puede satisfacer las dos condiciones identificadas por Duarte. Mediante esta "procedimentalización” de la regla, su antecedente sí establece condiciones necesarias y suficientes para la corrección de la decisión (la que puede, entonces, ser presentada como siguiéndose deductivamente de ella), sin que por ello se equiparen condiciones excepcionales y ordinarias (con esta formulación no es necesario probar que un candidato no es dirigente sindical para que pueda ocupar el escaño, pero sí que este ha obtenido la mayoría de los votos).

Según argumenta Duarte, esta "procedimentalización” de las reglas aplicables resulta inevitable para poder dar cuenta de la diferencia entre circunstancias ordinarias

\footnotetext{
${ }^{44}$ DuARte, 2015a, pp. 53 y ss.

45 Kelsen, 2005, pp. 236 y ss.

46 Kelsen, 2005, p. 239; 1944, p. 218 y 1957, p. 252.

${ }^{47}$ Duarte, 2015a, p. 54; y 2013. Al respecto, véase también Sartor, 1994, p. 189.
} 
y excepcionales. Si estas últimas operan derrotando una conclusión que sería correctamente alcanzada por la verificación de las circunstancias ordinarias, sin que por ello su no verificación deba ser tenida como condición necesaria para la corrección de dicha conclusión, entonces no sería posible ofrecer una representación puramente "substantiva" de ellas, i.e., que no haga referencia a lo que es "probado": debido a que "no probado que E" -el antecedente de la regla procedimentalizada de Duarte- es compatible con "no probado que no-E", entonces "no-E" no podría corresponder al supuesto hecho que “al nivel 'substantivo', se corresponde con la situación 'procedimental' en la que no se prueba" la circunstancia excepcional ${ }^{48}$. En otras palabras, de adoptarse una formulación sustantiva en que el antecedente de la regla es simplemente "si O y no-E, entonces C", entonces en caso de no probarse ni "E" ni "no-E”, no podría arribarse correctamente a $\mathrm{C}$, y con ello E ya no es una circunstancia excepcional que derrota una conclusión, sino que un elemento negativo ordinario.

No obstante ser exitoso en combinar ambos aspectos, este modelo trae consigo varias dificultades que hacen desaconsejable su adopción ${ }^{49}$. La principal de ellas ${ }^{50}$ es adecuadamente capturada por el argumento wittgensteiniano relativo al regreso de las reglas $^{51}$. Al entender que el antecedente de la regla debe dar cuenta de que, en un contexto judicial, la presencia de una circunstancia consiste en que ella sea probada conforme con otras reglas, y que debe hacerlo explícitamente mediante la inclusión del transitivo "probado", la tesis de Duarte queda comprometida a una sucesión infinita de reglas: en la medida que toda regla puede ser correcta o incorrectamente aplicada ${ }^{52}$, defender la necesidad de dar cuenta de las condiciones para la corrección de una aplicación en el contenido mismo de la regla a ser aplicada, supone exigir, para cada regla, una referencia explícita a nuevas reglas. Esto es advertido al atender a las reglas de prueba de las que dependería que dicha circunstancia se tenga por probada: ellas mismas tendrán un antecedente de hecho cuya "presencia" en un contexto judicial también dependería de que aquel fuera "probado" conforme con otras reglas. Así, la regla relativa a la fuerza probatoria de las declaraciones de testigos contestes no debería ser formulada en términos de "si dos o más testigos están contestes en el hecho y..., entonces se constituye plena

48 Duarte, 2015 a, pp. 63 y 123.

${ }^{49}$ Véanse las tres primeras objeciones levantadas por SARTOR, 1994, p. 188.

50 Además del problema de regreso, la procedimentalización de las reglas parece difícil de conciliar con los distintos estándares justificatorios que subyacerían a reglas sustantivas y procedimentales. Así, y a modo de ejemplo, mientras que las reglas procedimentales responden a diversos criterios de legitimidad (LAudan, 2006, p. 2. Acerca de la relación entre el proceso judicial y la verdad: Ho, 2008, Capítulo II), en un derecho penal sometido al principio de culpabilidad es el haber matado estando en posición de haber omitido matar (MAÑALICH, 2014a, p. 27), y no el que se pruebe que se ha matado, lo que legitima la sanción penal. De esta forma, la regla (sustantiva) “quien mate será castigado" se justifica en tanto condiciona la imposición de su consecuencia respecto de un determinado sujeto a que este haya cometido la ofensa que se le imputa (RAwLS, 1999, 74); pero dicha justificación no puede ser extendida a la regla procedimentalizada.

51 Wittgenstein, 1958, § 198-201.

52 Wittgenstein, 1958, § 198. Aplicando dicho argumento al razonamiento legal: Brandom, 2014, p. 21. 
prueba de ese hecho" -formularla de esta manera supondría que la verificación de su antecedente consiste simplemente en que "sea el caso" que dos testigos estén contestes en un hecho y no, como sugiere Duarte, en que ello fuese probado "de acuerdo a ciertas reglas y procedimientos" 53 - Para dar cuenta de esto último, el antecedente de la regla tendría que reformularse haciendo referencia a que "se pruebe" que dos testigos están contestes, antecedente cuya satisfacción requeriría a su vez una segunda regla de prueba y así sucesivamente ${ }^{54}$. Al requerir de una secuencia infinita de reglas (explícitas), el antecedente de la regla procedimentalizada no puede ser "probado conforme reglas", sino que simplemente tendrá que "ser el caso" que aquel se verifica -justamente lo que Duarte buscaba evitar-.

\section{UNA APROXIMACIÓN MATERIAL}

Conforme con camino recorrido hasta ahora, los tres modelos examinados aparecen como intentos insatisfactorios por dibujar la línea divisoria entre reglas y excepciones. Avanzar hacia una nueva articulación de la relación regla/excepción requiere en este punto dar un paso atrás y dirigir nuevamente la atención al descartado modelo nodeductivista, en particular a su variante conflictual. Hacerlo nos permitirá apreciar que la apariencia recién descrita es falsa: la objeción que Duarte levanta contra este descansa en un entendimiento "formalista" de las relaciones inferenciales a partir de la cuales se busca arribar a conclusiones correctas conforme con el derecho. Abandonando esa aproximación, y reemplazando por una "material”, será dicho modelo el que servirá de base para dibujar una línea nueva, capaz de sortear los constreñimientos examinados y explicar el rol desempeñado por las formulaciones de las reglas procedimentalizadas propuestas por Duarte.

Este último ha argumentado, en contra del modelo no-deductivista, que aquel ha de terminar comprometido con la misma conclusión que es explícitamente defendida por el modelo incorporacionista: ya que "ser dirigente sindical" constituye una condición suficiente para que la conclusión establecida por la regla no pueda correctamente ser alcanzada; entonces la negación de dicha circunstancias habrá de constituir, tal como "ser el candidato más votado", una condición necesaria para la corrección de la conclusión en cuestión.

Este movimiento, que va desde una premisa suficiente para la derrota de una conclusión a la premisa inversa como necesaria para ella, es el que debe ser rechazado: que una conclusión no pueda ser defendida en caso de sumarse a la inferencia una determinada premisa, no significa que la negación de esa premisa deba ser sumada a la inferencia para arribar a la conclusión - no en la medida que la corrección de la conclusión en la inferencia original pueda ser defendida atendiendo solo al significado de las premisas involucradas, es decir, no en la medida que la inferencia sea "materialmente correcta"-.

\footnotetext{
53 Duarte, 2015 a, p. 53.

${ }^{54}$ BRANDOM, 1994, pp. 18 y ss.
} 
Conforme con la semántica inferencial de Robert Brandom, la corrección de estas últimas, a diferencia de las inferencias formalmente válidas, descansa no en su forma sino en su contenido: lo que garantiza la corrección del movimiento es el contenido proposicional de las afirmaciones que sirven como premisas ${ }^{55}$. De este modo, y en tanto inferencias materiales, el aspecto crucial a ser considerado corresponde al significado de las premisas que configuran la inferencia, el que se encuentra constituido por el rol que, en la práctica discursiva ordinaria, cada afirmación juega tanto como razón en favor de otras afirmaciones y como demanda de otras afirmaciones en tanto razones a su favor; es decir, como premisa de la que se siguen materialmente ciertas conclusiones y como conclusión que se sigue materialmente de ciertas premisas ${ }^{56}$. Así, y aun cuando para su validez formal sería necesario sumar una premisa mayor, la inferencia desde "Santiago está al sur de Arica" a "Arica está al norte de Santiago" es materialmente correcta en consideración al significado de las expresiones involucradas ${ }^{57}$ : el hablante que se encuentre "comprometido" con una de las afirmaciones, se compromete por ello también con la otra ${ }^{58}$. Igualmente, la inferencia desde "este es un fósforo en buen estado" a "si lo golpeo prenderá" es correcta sin necesidad de sumar como premisa el condicional "si un fósforo está en buen estado, entonces prenderá"59: es parte del significado de la primera proposición el que ella sirve, en el juego social normativamente estructurado de dar y pedir razones, como razón para arribar a la segunda.

Vista como inferencia material, la inferencia es correcta. Pero también es derrotable: el fósforo no prenderá, por ejemplo, si se encuentra en campo electromagnético suficientemente fuerte ${ }^{60}$. De la misma manera, no será posible concluir que "debo abrir mi paraguas" a partir de la premisa "está lloviendo" si no deseo mantenerme seco. La posibilidad de echar abajo las inferencias mediante la adición de nuevas premisas podría ser tomada como señal de su incorrección: solo si se suma "deseo mantenerme seco" el movimiento sería correcto. Es la defensa de esta última idea la que caracteriza al "formalismo monotónico" 61 : la corrección de una inferencia depende de su forma, siendo necesario para alcanzar dicha corrección que se incluyan aquellas premisas que garanticen la conclusión incluso en caso de sumarse nuevos compromisos - deseos en el caso del razonamiento práctico y condicionales en el teórico-.

Ante este reclamo formalista es posible replicar que aquel no solo resulta difícil de conciliar con la evidente falta de necesidad de esgrimir dichas premisas faltantes durante la práctica discursiva ordinaria, en la que reconocemos a que "esté lloviendo" como razón para "abrir el paraguas", y conforme con ello quien afirma que una ciudad esté al sur de otra se compromete (virtud de esa afirmación) con que esa otra ciudad

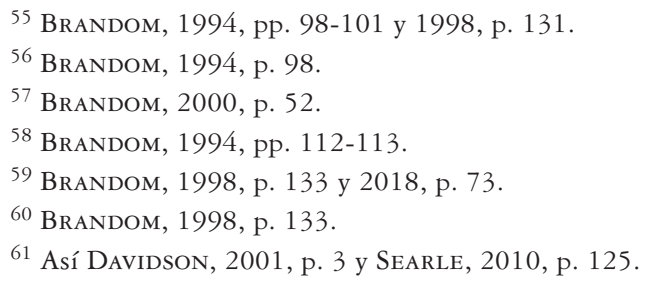


está al norte de la primera. Además, pierde de vista que la noción misma de inferencia "formalmente válida" es definible de manera natural a partir de la noción de inferencia "materialmente correcta" ${ }^{2}$. Defender que una premisa extra debe ser sumada para la corrección de la inferencia debe, además y crucialmente, enfrentar el problema de identificar cuál sería la premisa necesaria para sostener dicha corrección: aunque se incluya la premisa "el fósforo no se encuentra en un campo electromagnético", la conclusión de que este prenderá igualmente será derrotada si se vacía de oxígeno el ambiente que lo rodea. Y aunque se sumase que "el ambiente que rodea al fósforo contiene suficiente oxígeno”, sería de todas formas posible encontrar nuevas premisas que igualmente obstaculizarían el movimiento hasta la conclusión en cuestión. De esta forma, en vez de entender que sumar una cláusula ceteris paribus (cuyo contenido resultaría imposible de especificar) permitiría configurar a una inferencia monotónica, la necesidad de recurrir a aquella puede más adecuadamente ser comprendida como reconocimiento del carácter no-monotónico del razonamiento correcto en el discurso ordinario ${ }^{63}$. Así, que en caso de "desear mojarme" yo no pueda concluir que "debo abrir mi paraguas" en razón de que "está lloviendo", no muestra que para poder arribar a esa conclusión sea necesario incluir como premisa una cuyo contenido se corresponda a la negación del contenido de la premisa que echaría abajo la conclusión, sino que muestra que la inferencia, en tanto materialmente correcta (aunque no formalmente válida), es no-monotónica ${ }^{64}$.

\section{El modelo conflictual defendido}

Una vez que, contra el formalismo monotónico, se adopta una aproximación material no-monotónica, la variante conflictual del modelo no-deductivista no degenera en incorporacionismo. Para hacer esto explícito, tomemos como reglas relevantes las siguientes:

R1: "El candidato más votado ocupará el escaño respectivo".

R2: "Los dirigentes sindicales no podrán ocupar ningún escaño de elección popular".

R3: "Cuando R2 sea aplicable, R1 no lo será”.

El seguimiento de estas reglas efectivamente entraña que de verificarse el antecedente de R2 la conclusión de R1 no puede ser alcanzada. Así, si el juez suma como premisa que un candidato $\mathrm{J}$ es dirigente sindical, ello basta para que no pueda concluir que J ocupará el escaño respectivo -el compromiso con la primera precluye el derecho del hablante a comprometerse con la segunda ${ }^{65}$. Pero de ello solo se sigue que es necesario sumar como premisa que "J no es dirigente sindical" para poder concluir que "J ocupará

\footnotetext{
62 BRANDOM, 2000, p. 55.

${ }^{63}$ BRANDOM, 2018, p. 73

${ }^{64}$ BRANDOM, 1998, p. 132.

${ }^{65}$ Entre ambos compromisos existiría una relación de incompatibilidad material, BRANDOM, 1994, p. 169.
} 
el escaño respectivo" en caso de adoptarse la aproximación formalista monotónica ${ }^{66}$. Desde una perspectiva material, en cambio, para arribar a dicha conclusión basta que las premisas de la inferencia sean provistas por las tres reglas indicadas y solo una más: "J es el candidato más votado". Si bien -en tanto conclusión de una inferencia materialella podrá ser derrotada en caso de sumarse como premisa una que afirme respecto de $\mathrm{J}$ la satisfacción del estado especificado en el antecedente de R2, justamente -en tanto inferencia material- esto último no entraña tener a la no verificación del antecedente de R2 como condición necesaria para la corrección de la conclusión establecida en R1.

Vista materialmente y contra Duarte, la reconstrucción conflictual no equipara la verificación de circunstancias ordinarias (especificadas en R1) con la no verificación de las circunstancias excepcionales (especificadas en R2): el reconocimiento de ambas reglas potencialmente en conflicto determina que la no verificación de la circunstancia excepcional no cuenta como condición necesaria para arribar correctamente a conclusión establecida por R1, aun cuando su verificación sí constituye una condición suficiente para derrotarla.

Lo anterior permite mostrar que el modelo conflictual puede sortear no solo el primero, sino que también el segundo de los constreñimientos identificados al inicio de este trabajo. Para ello es necesario ofrecer un sentido diverso a la relación entre el antecedente de la regla a ser aplicada para la decisión del caso y la prueba de las circunstancias comprendidas en dicho antecedente. Conforme con el argumento defendido en la sección anterior, el problema fundamental del modelo procedimentalizado radica en su incapacidad de salvar el argumento relativo al regreso de las reglas. En contra de aquel, mantenido una formulación "sustantiva" de las reglas a ser aplicadas para la decisión del caso y entendiendo que las reglas de prueba fijan las condiciones bajo las cuales se prueba la satisfacción de las descripciones constitutivas de los supuestos de hecho de las primeras ${ }^{67}$, es posible entender esta exigencia de manera diversa. La inclusión, como premisa menor del silogismo, de una proposición en la que se afirma la satisfacción de la descripción constitutiva del antecedente de hecho de la regla, se encuentra condicionada a que tal proposición sea "probada conforme a reglas" y no a que ella sea "verdadera"68. Bajo esta comprensión, que esté "presente" la circunstancia referida por la regla sustantiva sigue dependiendo de que ella sea probada, pero ello es así en atención a la existencia de reglas de prueba referidas a las primeras, cuya correcta aplicación no necesita depender de subsecuentes reglas explícitas -que se trate de un contexto judicial no entraña que los antecedentes de hechos de todas las reglas a ser aplicadas en dicho contexto deban probarse conforme a reglas-. La necesidad de prueba, entonces, no se expresa en una "procedimentalización" de las reglas a ser aplicadas ni se extiende a todas las reglas aplicables en el contexto judicial, sino que en la identificación de las condiciones bajo las que puede incluirse una premisa que afirme la "presencia"

66 Así: Rodríguez y SuCAR, 1998, pp. 416-417.

${ }^{67}$ En un sentido similar: Bulygin, 1985, p. 163; Ferrer, 2005, p. 49 y 2006, p. 308.

${ }^{68}$ Esto supone entender que tal proposición puede ser probada aunque ella no sea verdadera, FERrER, 2005, p. 31 y 2006, p. 306. 
de la circunstancia comprendida su antecedente: respecto de un silogismo cuya premisa mayor sea provista por R1 o R2, solo podrá sumarse como premisa menor que "J es el candidato más votado" o que "J es dirigente sindical" si dichas proposiciones han sido probadas, con independencia de si acaso estas proposiciones corresponden a "hechos".

Si el argumento anterior es correcto, entonces, sin necesidad de procedimentalizar las reglas, sino que en consideración al trío de reglas identificado ${ }^{69}$, es posible superar ambos constreñimientos: conforme con estas últimas -y tal como en el modelo de Duarte- que "se pruebe que J es el candidato más votado" y que "no se pruebe que J es dirigente sindical" constituyen condiciones necesarias y suficientes para arribar a la conclusión de R1, condiciones que expresan las diferentes formas de operación que exhiben excepciones y elementos ordinarios, y cuya verificación no genera conflicto normativo alguno (por cuanto bajo ese resultado probatorio $^{70} \mathrm{R} 2$ no resulta internamente aplicable). Ya que la conclusión conforme a R1 se alcanza bajo las mismas condiciones de las que dependería la corrección de tal decisión conforme con el modelo procedimentalizado, el modelo conflictual permite tanto reconocer la diferencia en la forma de operación de los antecedentes de R1 y R2; como especificar el set de condiciones necesarias y suficientes para arribar correctamente a la conclusión establecida por $\mathrm{R} 1^{71}$.

\section{El modelo conflictual superado}

La revalidación del modelo conflictual no agota el redimiendo de la aproximación material acá defendida. Prestar atención la semántica inferencial en la que descansa el análisis de las relaciones materiales entre los contenidos proposicionales de las reglas en conflicto, permite además abordar exitosamente una pregunta que hasta ahora no ha sido formulada, y en cuya respuesta se encuentra la clave para ofrecer una reconstrucción de la relación regla/excepción que resulte no solo más simple -por requerir para la desactivación del conflicto menos premisas de las que son utilizadas por los modelos hasta ahora considerados-, sino que también de mayor poder explicativo -porque explicita el rol que juegan esos materiales extras-. A saber, ¿de qué depende que una circunstancia sea excepcional vis à vis una regla? En particular y respecto de R1 y R2, ¿por qué asumimos que ser dirigente sindical triunfa por sobre ser el candidato más votado y no viceversa? Responder esta pregunta desde una aproximación material permitirá mostrar cómo, ante un conflicto normativo generado por la conjunta aplicabilidad de R1 y R2, no es necesario ni invocar reglas de preferencia ni alterar las premisas originales para arribar materialmente a la conclusión jurídicamente correcta.

${ }^{69} \mathrm{El}$ argumento acá defendido no es uno relativo a la individuación de reglas, por el contrario, presupone que las reglas han sido ya individuadas. No obstante, la defensa del modelo conflictual sí muestra que no es necesario individuar reglas de forma tal que ellas no puedan generar un conflicto normativo.

70 Acerca de los distintos sentidos de "prueba”, véase Ferrer, 2005, p. 27.

${ }^{71}$ Que, debido a este set de reglas, sea posible identificar condiciones necesarias y suficientes para una decisión, no obsta a una comprensión del razonamiento legal como mayoritariamente "probativo", BRANDOM, 2014, p. 20. 


\section{a) Distinguiendo entre reglas y excepciones}

Una respuesta que descanse en la justicia material del resultado es ciertamente plausible en relación con la legítima defensa y el homicidio, pero no si se trata de R1 y R2 o de otras excepciones que eximan de pena en consideración a la raza de la víctima o impidan acceder a ciertas posiciones en consideración al estatus económico del candidato. Por otra parte, ya que conforme con la formulación propuesta de las reglas, ellas se encuentran en relación de interferencia, respecto de ninguno de estos ejemplos podría recurrirse al principio de especialidad para identificar las excepciones ${ }^{72}$. Tampoco resultaría mínimamente satisfactoria una explicación que intentara descansar en la "fineza del lenguaje”, de forma tal que las excepciones pudieran reconocerse en consideración a que ellas permiten identificar circunstancias en las que la aplicación de la regla resulta contraria a su finalidad, pero que no pueden ser excluidas por la regla misma en atención a que el lenguaje en el que ella se formula no "contiene una palabra o frase familiar que excluya lo que los redactores desean excluir del ámbito de la regla"73.

Una cuarta alternativa es ofrecida por el modelo procedimentalizado. Conforme con este, la distribución de las categorías "excepción” y "elementos ordinarios" depende a su vez de la distribución de la carga de la prueba, de forma tal que serán excepciones aquellas circunstancias que "no deben ser probadas" para alcanzar la conclusión en cuestión, mientras que corresponderán a elementos ordinarios aquellos que "deben ser probados" para arribar a aquella. Así, solo una vez que el legislador determina que la carga de probar la legítima defensa corresponde al imputado, y que la carga de probar la ausencia de consentimiento respecto del tipo de violación corresponde al persecutor, podemos afirmar que la primera es una excepción y la segunda no ${ }^{74}$. De ello se sigue que una regla que distribuya la carga de la prueba respecto de un set de circunstancias en consideración al carácter excepcional u ordinario que ellas exhiban ${ }^{75}$, resulta "imposible de aplicar", por "poner de cabeza la prioridad lógica” de la primera respecto de la segunda ${ }^{76}$.

El punto de partida para profundizar en una aproximación material al problema consiste en notar que hay algo particularmente contraintuitivo en esta solución. Para advertirlo, resulta conveniente comparar los casos hasta ahora examinados con el eventual conflicto normativo que se generaría por la simultánea aplicabilidad de una regla que castigase con pena de muerte a quien mutile y de otra que castigase con presidio perpetuo efectivo a quien viole ${ }^{77}$. Si atendemos solo a las relaciones lógicas entre los

${ }^{72}$ Más aún, la regla de legítima defensa no tiene porque ser especial ni siquiera respecto de la conjunción de la totalidad de las reglas que establecen los tipos penales. Para aproximación material a la relación de especialidad, véase: SzCZARANSKI, 2021.

73 SCHAuer, 1991, p. 874. Tal explicación ignoraría que semejante "falta de fineza" habrá de afectar tanto a los términos empleados por la "regla principal" como a la disposición que consagre la excepción.

${ }^{74}$ Duarte, 2015 a, p. 133.

75 Al respecto, véase MacCoRMick, 2005, pp. 244 y ss.

76 Duarte, 2015 a, p. 133.

${ }^{77}$ Kelsen, 1973, p. 234 
antecedentes y consecuentes de las reglas, este último caso es formalmente idéntico a aquel generado por R1 y R2, y también al que se configuraría en un caso de la legítima defensa en donde las reglas involucradas se formulasen de la siguiente manera:

R3: "Quien mate a otro sufrirá una pena de 10 años de privación de libertad".

R4: "Quien actúe en legítima defensa no habrá de sufrir pena alguna”.

En estos tres supuestos y de conformidad al modelo procedimentalizado, la identificación de la solución correcta requeriría contar con una nueva premisa, un criterio externo a las reglas que permita reformularlas. Y es en relación con la necesidad de incluir premisas adicionales que el modelo conflictual resulta equivalente al propuesto por Duarte: allí también será necesario recurrir a un criterio externo a las reglas involucradas (una regla de preferencia) a efectos de desplazar a una de ellas. En esta medida ambas aproximaciones expresan un compromiso con una aproximación formal: tanto en relación con $\mathrm{R} 1$ y $\mathrm{R} 2$, como con $\mathrm{R} 3$ y $\mathrm{R} 4$, y al igual que en el caso de mutilaciones y violación, no sería posible reconocer una asimetría que justifique un trato diferenciado sino hasta que se sume una nueva premisa relativa a la distribución probatoria o a la preferencia entre ellas ${ }^{78}$.

No obstante lo anterior, mientras que no encontramos una respuesta obvia para el caso de las sanciones incompatibles, sí parece natural reconocer que "ser dirigente sindical" y "actuar en legítima defensa" han de ser tomadas como las circunstancias excepcionales, incluso antes de la inclusión de una regla explícita que rompa la simetría. A pesar de compartir una misma forma, respecto de estos últimos no parece haber dificultad para identificar la conclusión correcta -el problema radica, más bien, en "explicar" cómo se arriba a ella-. A diferencia de los modelos formalistas, adoptar una aproximación material exige atender a los contenidos de las reglas involucradas con el fin de buscar una diferencia entre los conflictos que sean relevantes para su solución, pero invisibilizada por la homogeneidad de sus formas. Esa diferencia consiste en la dependencia práctica que caracteriza a las reglas R2, sobre dirigentes sindicales, y R4, sobre legítima defensa, en

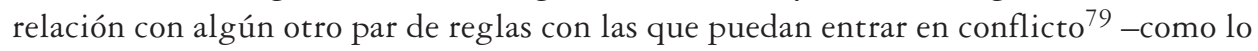
son R1 y R3, respectivamente-. Ya que “ocupar el escaño respectivo” corresponde a una posición institucional cuya instanciación depende de la satisfacción de las condiciones enunciadas por alguna regla que cree el respectivo estatus-función ${ }^{80}$, en ausencia de una regla constitutiva que resulte aplicable a un caso -como R1- no es necesario recurrir a R2 para arribar a la conclusión que ella establece. Así, respecto de un sujeto que no obtiene la mayoría de los votos, que aquel "sea dirigente sindical” no juega ningún rol a efectos de concluir que dicho sujeto "no podrá ocupar ningún escaño de elección popular”. Lo

\footnotetext{
${ }^{78}$ En un sentido similar: Ross, 1959, p. 130.

${ }^{79}$ La noción de "dependencia práctica" empleada para desactivar conflictos normativos (aunque sin recurrir a la default/challenge estructure con la que es complementada en el presente trabajo), es desarrollada en: SZCZARANSKI, en revisión.

${ }^{80}$ SEARLe, 2010, p. 167.
} 
mismo es el caso en relación con R3 y R4: solo resulta pertinente aducir la circunstancia consistente en haber actuado en legítima defensa para concluir que un sujeto "no habrá de sufrir pena alguna" en la medida que se cuente también con una regla como R4, cuya aplicabilidad al caso permita concluir que dicho sujeto sí habrá de sufrir alguna pena. En otros términos: mientras que matar a otro y ser el candidato más votado constituyen condiciones necesarias para arribar a las conclusiones de las reglas R1 y R3; actuar en legítima defensa y ser dirigente sindical no son, en principio, circunstancias necesarias para arribar a las conclusiones establecidas por R2 y R4.

La importancia de lo anterior admite ser explicitada en términos de la procedencia de justificaciones relativas a afirmaciones a las que los hablantes tienen, por defecto, derecho: es parte de la estructura de la práctica discursiva ordinaria que al realizar una afirmación el hablante asume la responsabilidad de vindicarla ${ }^{81}$, es decir, de ofrecer nuevas afirmaciones que le sirvan como premisas. Aunque lo anterior vale por regla general, en la práctica discursiva ordinaria no toda afirmación necesita ser justificada, habiendo algunas a las que todo hablante tiene derecho sin necesidad, en principio, de aducir nuevas afirmaciones que le sirvan de premisas. Así, ya que prima facie se tiene derecho a afirmar que -por ejemplo- "han habido perros negros", ofrecer una justificación para dicha afirmación resulta inadecuado en ausencia de afirmaciones justificadas que la desafíen ${ }^{82}$. Solo si se suman nuevas premisas que ceden en contra de la afirmación a la que por defecto se tiene derecho surge la responsabilidad de esgrimir una o más premisas que sirva de justificación a esta última. De esta forma, las afirmaciones que justifican una afirmación prima facie o default son inferencialmente inertes tanto en ausencia de premisas incompatibles como si, habiéndolas, no logran derrotarlas. De esta observación es posible extraer una consecuencia fundamental: porque la responsabilidad justificatoria de vindicar compromisos se encuentra condicionada a que estos últimos sean desafiados justificadamente, entonces el rol de las afirmaciones que sirven como razones en favor de afirmaciones default -i.e., que sirven como premisas de las que estas últimas se siguen- se desempeña solo dentro de una red de razones que sirven para arribar a conclusiones incompatibles, consistiendo tal rol, precisamente, en derrotar a estas últimas. Así, y debido al carácter no-monotónico de las inferencias materiales, es posible construir una estructura jerarquizada de razones ${ }^{83}$, gobernada por los contenidos de las premisas involucradas, en las que una afirmación default es derrotada por premisas que la desafían justificadamente, pudiendo estas a su vez ser derrotadas por aquellas que, vindicando la responsabilidad justificatoria generada por la formulación del desafío, sirven para reafirmar la conclusión original.

Esta Default/Challenge Structure provee la clave para identificar, sin necesidad de recurrir a nuevas premisas, cuál de las reglas en conflicto ha de primar a efectos de determinar la conclusión que habrá de corresponder a la decisión del caso: porque el

\footnotetext{
${ }^{81}$ BRANDOM, 1994, p. 171.

82 BRANDOM, 1994, p. 176.

${ }^{83}$ BRANDOM, 1998, p. 133.
} 
estatus-función cuya configuración se ve bloqueada por la verificación los antecedentes de R2 y R4 solo se instancia en caso que se satisfagan las condiciones enunciadas por las reglas constitutivas que creen los estatus en cuestión ${ }^{84}$, las primeras solo resultan pertinentes a efectos de arribar a las conclusiones que ellas establecen en tanto que al caso al que ellas son aplicables le sean también aplicables las segundas ${ }^{85}$. Es por esta razón que $\mathrm{R} 2$ y $\mathrm{R} 4$ proveen premisas para (re)afirmar conclusiones que corresponden a afirmaciones default, siendo entonces la estructura misma del juego de dar y pedir razones la que determina que aquellas jueguen el rol de premisas que han de derrotar a otras reglas, como R1 y R3, que desafían sus consecuencias.

De esta forma, es contrastándolas con los estatus por default que resulta posible advertir, detrás de la simetría formal entre las reglas en conflicto, una asimetría entre sus contenidos que gobierna la distribución de los roles entre ellas: mientras que las reglas R1 y R3 desafían ese estatus, R2 y R4 lo justifican, y es por ello que la ubicación de estas últimas en una estructura jerarquizada de razones se encuentra, en principio, por sobre las primeras - en tanto que a sus consecuencias se tiene derecho sin necesidad de esgrimirlas, el rol que corresponde conforme a la práctica discursiva ordinaria a las reglas respecto de dirigentes sindicales y legítima defensa es el de derrotar otras reglas cuyas consecuencias resulten incompatibles con las afirmaciones prima facie a las que aquellas sirven de premisa-. He aquí la razón subyacente a nuestra natural disposición a diferenciar entre estos casos y aquel en que dos reglas disponen sanciones incompatibles. En este último, ambas reglas desafían la conclusión a la que, en ausencia de otras reglas aplicables, tenemos derecho sin necesidad de ofrecer justificación (que nadie habrá de sufrir pena alguna), por lo que no es posible recurrir a un estatus por default para distribuir roles entre ellas. En cambio, y en contra del modelo conflictual, de verificarse esta asimetría entre los consecuentes, respecto de una inferencia material cuyas premisas estén provistas por las reglas en conflicto y por una descripción del caso a ser decidido conforme a la cual los antecedentes de ambas reglas se ven instanciados, no es necesario aducir una premisa extra provista por una regla de preferencia para arribar a la solución correcta: la inferencia, tal como está, tendrá como conclusión materialmente correcta a aquella que corresponda a la establecida por la regla que afirme el estatus por default.

Corolario de las consideraciones precedentes es que resulta posible caracterizar la relación regla/excepción en términos de un par de reglas tales que una, al constituir una premisa para justificar una afirmación a la que se tiene derecho por defecto, resulta inferencialmente dependiente de (y por ello derrota a) aquella que desafíe la afirmación que la primera vindica.

${ }^{84}$ MACCORMICK, 1986, p. 55.

${ }^{85}$ Es en este sentido en que es correcta la afirmación de Williams, 1988, p. 279: an exception distinct from a rule is inconceivable. 


\section{b) Corrección material y explicitación formal}

Que en casos de dependencia práctica no sea necesario procedimentalizar las premisas, permite por último atribuir un rol "expresivo" a la formulación procedimentalizada propuesta por Duarte. Ella buscaba dotar de validez formal a la inferencia por la que se arriba a la conclusión correcta en estos casos, garantizando así la corrección de la decisión. El argumento desarrollado en este trabajo ofrece una perspectiva distinta a la relación entre forma y contenido: en una aproximación material, la especificación de condiciones necesarias y suficientes no se asocia a la posibilidad de tener a una decisión como correcta, sino que constituye un esfuerzo por explicitar en forma deductiva la corrección del razonamiento material ${ }^{86}$. Así, mientras que lo que hacemos correctamente con las reglas en conflicto es arribar a una conclusión conforme con la regla independiente si es que su antecedente es probado y no se prueba el antecedente de la regla dependiente; el contenido proposicional de la regla procedimentalizada constituye una forma de expresar esa práctica, diciendo explícitamente que "si se prueba $\mathrm{O}$ y no se prueba E entonces C" - esto es, especificando parte de la habilidad que debe ser exhibida para realizar inferencias materiales correctas con las reglas en conflicto ${ }^{87}$-.

La importancia de poder identificar condiciones necesarias y suficientes para una decisión, de manera tal que las conclusiones legales puedan ser presentadas como siguiéndose deductivamente de alguna combinación de enunciados sobre reglas y sobre los hechos del caso, no radica entonces en asegurar la corrección de la decisión, sino que en hacer posible la explicitación de la corrección del razonamiento legal conforme con las inferencias materiales subyacentes. Para ello, y contra Duarte, no es necesario que las reglas a ser aplicadas hagan ellas mismas explícitas esas condiciones.

\section{BIBLIOGRAFÍA}

Alchourrón, Carlos y Bulygin, Eugenio, 1981: “The Expressive Conception of Norms”, en Hilpinen (ed.), New Studies in Deontic Logic, Dordrecht, Reidel Publishing, pp. 95-124.

Baker, G. P., 1977: "Defeasibility and Meaning”, en Hacker y Raz (eds.), Law, Morality, and Society, Oxford, Clarendon Press, pp. 26-57.

Bentham, Jeremy 1970: Of Laws in General, London, Athlone Press.

Brandom, Robert, 1994: Making it Explicit, Cambridge: Harvard University Press.

Brandom, Robert, 1998: “Actions, Norms and Practical Reasoning”, Philosophical Perspectives, Vol. 12.

Brandom, Robert, 2000: Articulating Reasons, Cambridge, Harvard University Press. Brandom, Robert, 2008: Between Saying and Doing, Oxford: Oxford University Press. Brandom, Robert, 2014: “A Hegelian Model of Legal Concept Determination”, en Hubbs y Lind (eds.), Pragmatism, Law and Language, New York, Londres, Routledge, pp. 19-39.

\footnotetext{
${ }^{86}$ BRANDOM, 2018, p. 9 y ss.

${ }^{87}$ Brandom, 1998, p. 132 y 2008, p. 9 y ss.
} 
Brandom, Robert, 2018: "From Logical Expressivism to Expressivist Logic", Philosophical Issues, Vol. 28, No 1 .

Bulygin, Eugenio y MendonCa, Daniel, 2005: Normas y Sistemas Normativos, Madrid, Marcial Pons. Bulygin, Eugenio, 1985: "Norms and Logic", Law and Philosophy, Vol. 4.

Davidson, Donald, 2001: Essays on Actions and Events, Oxford: Oxford University Press.

Duarte, Luis, 2013: "A Proof-Based Account of Legal Exceptions", Oxford Journal of Legal Studies, Vol. 33, $\mathrm{N}^{\circ} 1$.

DuArTe, Luis, 2015a: Allowing for Exceptions. A Theory of Defences and Defeasibility in Law, Oxford, Oxford University Press.

Duarte, Luis, 2015b: "Defining 'Defences", en A.A.V.V., Defences in Tort, London, Hart Publishing, pp. 35-52.

Duarte, Luis, 2019: "On the Legal Syllogism", en Plunkett, Shapiro y Toh (eds.), Dimensions of Normativity, Oxford, Oxford University Press, pp. 335-364.

Elhag, A. A. O., Breuker, J. A. P. J. y Brouwer, P. W., 2000: "On the Formal Analysis of Normative Conflicts”, Information E Communications Technology Law, Vol. 9, N 3 .

Ferrer, Jordi y Rodríguez, Jorge, 2011: Jerarquías Normativas y Dinámica de los Sistemas Jurídicos, Madrid, Marcial Pons.

Ferrer, Jordi, 2005: Prueba y Verdad en el Derecho, Madrid, Marcial Pons.

Ferrer, Jordi, 2006: "Legal Proof and Fact Finders' Beliefs", Legal Theory, Vol. 12.

Finkelstein, C.O., 1999: "When the Rule Swallows the Exception”, en Meyer (ed.), Rules and Reasoning, Oxford, Hard Publishing, pp. 147-175.

García, José, 2020: "La Noción de Derrotabilidad en Hart", Ius et Praxis, Año 26, No 2.

Guastini, Riccardo, 1999: Distinguiendo. Estudios de Teoría y Metateoría del Derecho, Barcelona, Gedisa.

Hamblin, C. L., 1972: "Quandaries and the Logic of Rules”, Journal of Philosophical Logic, Vol. 1, $\mathrm{N}^{\circ} 1$.

Hammer, H., 1987: "A Functional Taxonomy of Normative Conflict”, Law and Philosophy, Vol. 6, $\mathrm{N}^{\circ} 2$.

Hart, H. L. A., 1949: "The Adscription of Responsibility and Rights", Proceedings of the A ristotelian Society, Vol. 49.

Ho, H. L., 2008: A Philosophy of Evidence Law, Oxford, Oxford University Press.

Kelsen, Hans, 1944: "The Principle of Sovereign Equality of States as a Basis for International Organization", The Yale Law Journal, Vol. 53, No 2.

Kelsen, Hans, 1957: What is Justice?, Berkeley, University of California Press.

Kelsen, Hans, 1973: Essays in Legal and Moral Philosophy, Dordrecht, Reidel Publishing.

Kelsen, Hans, 1991: General Theory of Norms, Oxford, Clarendon Press.

Kelsen, Hans, 2005: Pure Theory of Law, New Jersey, The Lawbook Exchange.

Kelsen, Hans, 2006: General Theory of Law and State, New Brunswick, Transaction Publishers.

Laudan, Larry, 2006: Truth, Error and Criminal Law, New York, Cambridge University Press.

MacCormick, Neil, 1986: "Law as Institutional Fact", en MacCormick y Weinberger, An Institutional Theory of Law, Dordrecht, Reidel, pp. 49-76.

MacCormick, Neil, 1995: "Defeasibility in Law and Logic", A.A.V.V., Informatics and the Foundations of Legal Reasoning, Dordrecht, Kluwer, pp.99-117.

MacCormick, Neil, 2005: Rhetoric and the Rule of Law, Oxford, Oxford University Press.

Mackie, J. L., 1985: Persons and Values. Selected Papers. Volume II, Oxford, Clarendon Press.

Mañalich, Juan Pablo, 2014a: Norma, Causalidad y Acción, Madrid, Marcial Pons.

Mañalich, Juan Pablo, 2014b: "Normas Permisivas y Deberes de Tolerancia”, Revista Chilena de Derecho, Vol. 41, No 2. 
Miller, Leonard, 1956: "Rules and Exceptions", Ethics, Vol. 66, № 4.

Moore, Michael, 1989a: "Authority, Law and Razian Reasons”, Southern California Law Review, Vol. 62, $\mathrm{N}^{\circ} 3 \& 4$

Moore, Michael, 1989b: "Torture and the Balance of Evils", Israel Law Review, Vol. 23, № 2\&3.

Navarro, Pablo y Moreso, J. J., 1997: “Applicability and Effectiveness of Legal Norms”, Law and Philosophy, Vol. 16.

Navarro, Pablo; Orunesu, Claudina; Rodríguez, Jorge y Sucar, Germán, 2004: “Applicability of Legal Norms", Canadian Journal of Law and Jurisprudence, Vol. XVII, No 2.

Navarro, Pablo y Rodríguez, Jorge, 2014: Deontic Logic and Legal Systems, New York, Cambridge University Press.

Ortiz de Urbina, Íñigo, 2008: “De Moscas y Agresores Muertos”, InDret Penal, 3/2008.

RaWls, John, 1999: A Theory of Justice, Cambridge, Massachusetts, The Belknap Press.

RAz, Joseph, 1980: The Concept of a Legal System, Oxford, Clarendon Press.

Raz, Joseph, 1989: "Facing Up: A Reply", Southern California Law Review, Vol. 62, No $3 \& 4$.

Rodríguez, Jorge y Sucar, Germán, 1998: "Las Trampas de la Derrotabilidad”, Doxa, Vol. 2, Nº 1.

Rodríguez, Jorge, 1997: "La Derrotabilidad de las Normas Jurídicas”, Isonomía, No 6.

Ross, Alf, 1959: On Law and Justice, Berkeley, University of California Press.

SARtor, Giovanni, 1992: "Normative Conflicts in Legal Reasoning", Artificial Intelligence and Law, Vol. 1.

SARTor, Giovanni, 1994: "A Formal Model of Legal Argumentation”, Ratio Juris, Vol. 7, N 2.

SCHauer, Frederick, 1991: "Exceptions", The University of Chicago Law Review, Vol. 58, № 3.

Searle, John, 1987: "Prima Facie Obligations", en Raz (ed.), Practical Reasoning, Oxford, Oxford University Press, pp. 81-90.

Searle, John, 2010: Making the Social World, Oxford, Oxford University Press.

Stavropoulos, Nicos, 1996: Objectivity in Law, Oxford, Clarendon Press.

Stone, Julius, 1944: "Burden of Proof and the Judicial Process", Law Quarterly Review, Vol. 60, $\mathrm{N}^{\circ} 3$.

Stone, Julius, 1985: Precedent and Law, Sydney, Butterworths.

SzCZARANSKI, Federico, 2021: "Sobre el Rol Expresivo del principio ne bis in idem: Una Aproximación Inferencial al Concurso Aparente por Especialidad", en Maldonado (ed.), Concurso de Delitos. Problemas Fundamentales, Santiago, Tirant lo Blanch (de próxima publicación).

SzCZaranski, Federico (en revisión): "On Normative Redundancies and Conflicts: A Material Approach", Law and Philosophy.

Von Wright, G. H., 1963: Norm and Action, London, Routledge \& Kegan Paul.

Von Wright, G. H.,1991: “Is There a Logic of Norms?”, Ratio Juris, Vol. 4, No 3.

Von Wright, G. H.,1983: Practical Reason, Oxford, Basil Blackwell.

Waluchow, Wilfrid, 2012: "Defeasibility and Legal Positivism”, en Ferrer y Battista (eds.), The Logic of Legal Requirements, Oxford, Oxford University Press, pp. 254-267.

Weinberger, Ota, 1972: "Fundamental Problems of the Theory of Legal Reasoning", Archives for Philosophy of Law and Social Philosophy, Vol. 58, $\mathrm{N}^{\circ} 3$.

Williams, Glanville, 1982: "Offences and Defences", Legal Studies, Vol. 2, No 3.

Williams, Glanville, 1988: “The Logic of 'Exceptions”, The Cambridge Law Journal, Vol. 47, № 2.

Wittgenstein, Ludwig, 1958: Philosophical Investigations, Oxford, Basil Blackwell. 